\title{
Neuwahlen der Swissmedic Medicines Expert Committees
}

\section{Esther Wullimann}

Dr., wissenschaftliche Mitarbeiterin und Verantwortliche Expertenwesen, Abteilung Regulatory Operations and Development, Bereich Zulassung, Schweizerisches Heilmittelinstitut (Swissmedic)

\section{Bei der Zulassung, Marktüberwachung oder Bewilligung von Human- und Tierarz- neimitteln unterziehen die Swissmedic Medicines Expert Committees (SMEC) die wissenschaftliche Dokumentation der Zulassungsgesuche einer unabhängigen zweiten Prüfung.}

Wie alle international führenden Arzneimittelbehörden lässt sich auch das Schweizerische Heilmittelinstitut Swissmedic in wissenschaftlichen Fragen von externen Expertengremien beraten: dem Human Medicines Expert Committee (HMEC) für Humanarzneimittel und dem Veterinary Medicines Expert Committee (VMEC) für Tierarzneimittel. Die Zusammenarbeit mit externen Experten ist ein wichtiges Instrument, um die Qualität der Entscheide sicherzustellen. Der europäischen EMA stehen dafür verschiedene Scientific Advisory Groups zur Seite; die amerikanische Heilmittelbehörde FDA arbeitet eng mit ihren Advisory Committees zusammen.

\section{Die Zusammenarbeit mit externen Experten ist ein wichtiges Instrument, um die Qualität der Entscheide sicherzustellen.}

Die Swissmedic Medicines Expert Committees (SMEC) bestehen aus ordentlichen, ausserordentlichen und beratenden Mitgliedern und werden vom SwissmedicInstitutsrat für eine vierjährige Amtszeit gewählt. Ihre Expertinnen und Experten verfügen über ausgewiesene berufliche Qualifikationen und Expertisen in Medizin, Pharmazie oder Naturwissenschaften und haben breite Erfahrungen im klinischen Umfeld.

\section{Unabhängige Experten als zusätzliches qualitätssicherndes Element}

Swissmedic nimmt externe Expertisen hauptsächlich im Rahmen der Zulassung, Marktüberwachung und Bewilligung von Arzneimitteln und Transplantatprodukten sowie der Marktüberwachung von Medizinprodukten in Anspruch. Die Expertinnen und Experten validieren die intern erarbeiteten Evaluationsberichte (assessment reports) und leisten im kritischen Dialog mit den Swissmedic-Fachexperten einen wichtigen Beitrag zur objektiven wissenschaftlichen Entscheidfindung. Insbesondere das praxisnahe Wissen aus dem klinischen Alltag ist eine wesentliche Ergänzung zu hausinternen Fachkompetenzen.

Die Swissmedic Medicines Expert Committees (SMEC) haben in den Jahren 2017 bis 2019 insgesamt 233 Empfehlungen zur Zulassung und Anwendung von Arzneimitteln in der Schweiz abgegeben (205 HMEC, 28 VMEC). Sie leisten so einen unverzichtbaren Beitrag, um Schweizer Ärztinnen und Ärzten in ihrer täglichen Arbeit wirksame, sichere und qualitativ einwandfreie Arzneimittel zur Verfügung zu stellen.

Damit eine unabhängige Beratertätigkeit gewährleistet werden kann, erlässt der Swissmedic-Institutsrat den «Kodex zum Umgang mit Interessenkonflikten

Tabelle 1: Einbezug des Human Medicines Expert Committees (HMEC)

in die Begutachtungstätigkeit von Swissmedic in den Jahren 2017-2019.

\begin{tabular}{lllll}
\hline Tätigkeit HMEC & $\mathbf{2 0 1 7}$ & $\mathbf{2 0 1 8}$ & $\mathbf{2 0 1 9}$ & Total \\
\hline Anzahl Empfehlungen zu Gutheissung/Abweis & 73 & 71 & 61 & 205 \\
\hline Anzahl Einzelexpertisen & 24 & 33 & 23 & 80 \\
\hline Anzahl vollständige Evaluationen von Dossierteilen & 14 & 13 & 14 & 41 \\
\hline Total & $\mathbf{1 1 1}$ & $\mathbf{1 1 7}$ & $\mathbf{9 8}$ & $\mathbf{3 2 6}$
\end{tabular}




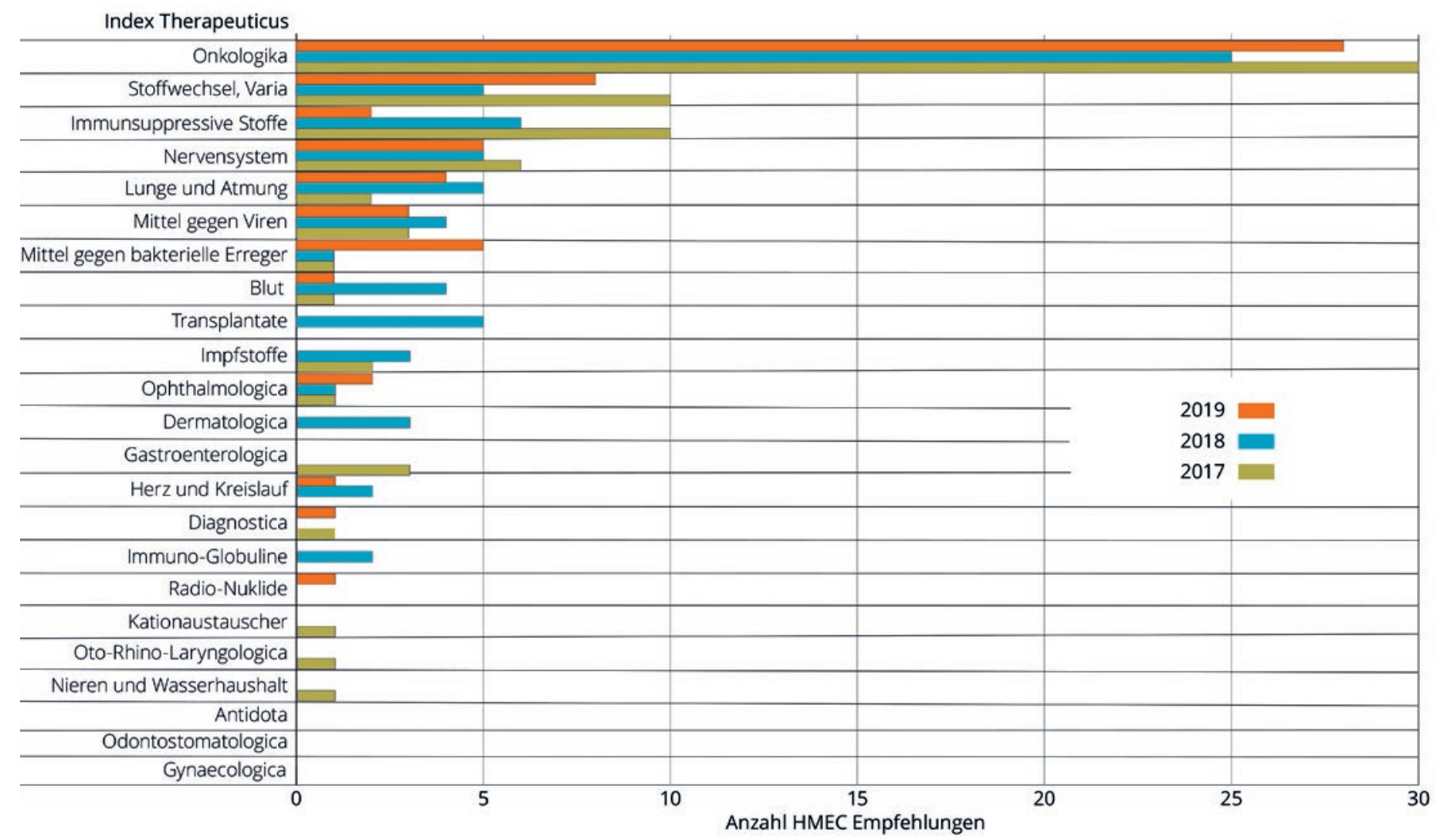

Tabelle 2: Anzahl HMEC Empfehlungen nach Anwendungsgebiet von 2017-2019.

für die Swissmedic Medicines Expert Committees». Zentrale Elemente darin sind die Definition der Interessenkonflikte, die Offenlegungspflichten und die Ausstandsregelung. Jedes SMEC-Mitglied deklariert jährlich seine aktuellen Interessenbindungen, und Swissmedic publiziert diese auf ihrer Internetseite. Wird fallweise ein Interessenkonflikt festgestellt, tritt der betroffene Experte in den Ausstand.

\section{Human Medicines Expert Committee (HMEC)}

Das HMEC umfasst rund 60 Mitglieder. Ein ordentliches Gremium bestehend aus Experten zur Inneren Medizin, (klinischen) Pharmakologie, Toxikologie, Onkologie, Neurologie und Infektiologie wird dabei durch eine Gruppe von ausserordentlichen und beratenden Experten unterstützt, welche eine breite Abdeckung der verschiedensten Fachgebiete sicherstellen. Neben Spezialisten in medizinischen Disziplinen wie der Kardiologie, Ophthalmologie oder Gastroenterologie runden Experten in Statistik, Phytopharmazie oder regulatorischer Toxikologie die vielseitige Kompetenz des HMEC ab.
Das ordentliche Gremium, bestehend aus neun Mitgliedern, trifft sich monatlich bei Swissmedic in Bern und erwägt die Wirksamkeit und Sicherheit von neu zur Zulassung beantragten innovativen Humanarzneimitteln und Indikationserweiterungen. Nach Präsentation der Experteneinschätzung erfolgt im Plenum eine kritische Diskussion zu Nutzen und Risiken, welche in einer Empfehlung an Swissmedic über Gutheissung oder Abweis der entsprechenden Anträge mündet. Ist die Beurteilung kontrovers, wird die Empfehlung in einer Abstimmung herbeigeführt. In den vergangenen Jahren hat sich gezeigt, dass die Swissmedic-Beurteilung und die HMEC-Empfehlung in den meisten Fällen übereinstimmen. Letztlich obliegt der finale Entscheid jedoch immer Swissmedic.

\section{Bei Bedarf übernehmen HMEC-Experten} die vollständige Evaluation von Teilen eines Zulassungsdossiers.

Die Erarbeitung von Empfehlungen zu Gutheissung oder Abweis von neuen aktiven Substanzen und Indikationserweiterungen stehen im Vordergrund der HMEC-Tätigkeiten. Zusätzlich werden sowohl ordentliche als auch ausserordentliche und beratende Experten 
punktuell zu spezifischen Fragestellungen, welche sich im Rahmen der Swissmedic-internen Begutachtung ergeben, beigezogen (Einzelexpertise). Bei Bedarf übernehmen HMEC-Experten zudem die vollständige Evaluation von Teilen eines Zulassungsdossiers. Je nach Fachgebiet des Experten kann es sich dabei um die Dokumentation der Klinik, Präklinik oder Qualität eines Arzneimittels handeln.

Die bearbeiteten Zulassungsdossiers decken ein breites Spektrum an Anwendungsgebieten ab. In den letzten Jahren zeichnet sich jedoch ein zunehmender Fokus auf Onkologika (inkl. Immun-Onkologika) ab. 83 der insgesamt 205 HMEC-Empfehlungen betrafen diese Arzneimittelgruppe, was einem Anteil von 40\% entspricht. Zum aktuellen Zeitpunkt kann davon ausgegangen werden, dass dieser Trend weiter anhält.

Ende 2020 läuft die 4-jährige Amtsperiode der SMEC aus, und die Gesamterneuerungswahlen für die Amtsperiode 2021-2024 stehen an. Swissmedic möchte die Gelegenheit nutzen, um die fachliche Kompetenz der
Gremien weiter zu stärken. In der Humanmedizin werden Experten insbesondere in den Fachgebieten Onkologie, Pneumologie, Gastroenterologie, Ophthalmologie, Gynäkologie, Urologie, Pädiatrie, Allergologie, Immunologie/Vakzinologie, Infektiologie/Virologie, Endokrinologie und klinische Pharmakologie gesucht.

\section{Das Wichtigste in Kürze}

- Externe Expertengremien unterstützen Swissmedic mit Wissen aus dem akademischen Bereich und der Praxis und leisten so einen wichtigen Beitrag, damit Schweizer Ärztinnen und Ärzten in ihrer täglichen Arbeit wirksame, sichere und qualitativ einwandfreie Arzneimittel zur Verfügung stehen.

- In den Jahren 2017 bis 2019 haben die Expertinnen und Experten insgesamt 233 Empfehlungen zur Neuzulassung und zu Indikationserweiterungen von Arzneimitteln in der Schweiz abgegeben.

- Bei den Humanarzneimitteln lag dabei der Fokus stark auf den Onkologika.

- Im Hinblick auf die Gesamterneuerungswahlen Ende 2020 sucht Swissmedic Kandidatinnen und Kandidaten.

\section{Unterhaltung und Spannung aus der Feder von Ärzten}
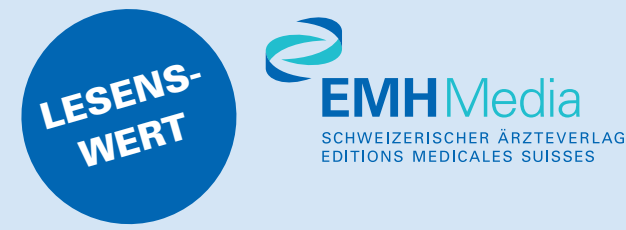

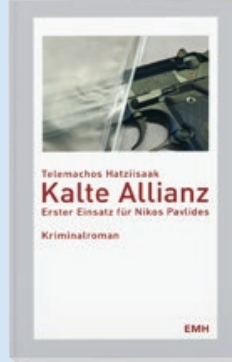

Kalte Allianz von Telemachos Hatziisaak

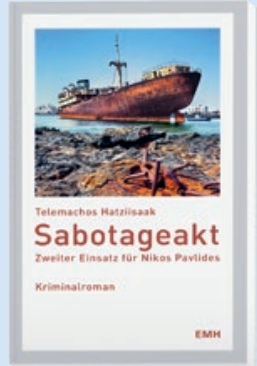

Sabotageakt von Telemachos Hatziisaak

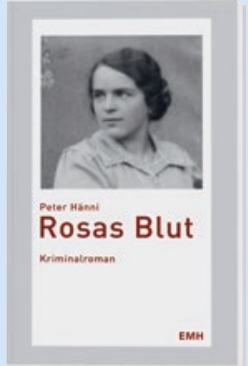

Rosas Blut von Peter Hänni

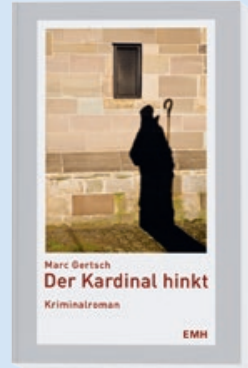

Der Kardinal hinkt von Marc Gertsch SCHWEIZERISCHER ÄRZTEVERLA
EDITIONS MEDICALES SUISSES
Bestellung

(Fax 06146785 76)

Ex. Kalte Allianz | Telemachos Hatziisaak | CHF 23.-

Ex. Sabotageakt | Telemachos Hatziisaak | CHF 23.-

Ex. Rosas Blut | Peter Hänni | CHF 21.50

Ex. Der Kardinal hinkt | Marc Gertsch | CHF 23.

Vorname / Name
Strasse / Nr.
PLZ / Ort
E-Mail
Datum

